\title{
Emendation of the genus Sphingomonas Yabuuchi et al. 1990 and junior objective synonymy of the species of three genera, Sphingobium, Novosphingobium and Sphingopyxis, in conjunction with Blastomonas ursincola
}

\footnotetext{
${ }^{1}$ Department of Microbiology, Gifu University School of Medicine, Tsukasa-machi 40, Gifu 500-8705, Japan

2 Japan Collection of Microorganisms, Institute of Physical and Chemical Research (RIKEN), Wakoshi, Saitama 351-0198, Japan

${ }^{3}$ Department of Host Defense, Osaka City University Graduate School of Medicine, 1-4-3 Asahimachi, Abeno-ku, Osaka 545-8585, Japan

${ }^{4}$ Institute of Skin Sciences, Club Cosmetics Co. Ltd, Ikoma-shi, Nara 630-0222, Japan

${ }^{5}$ Department of Virology, Osaka City University Graduate School of Medicine, 1-4-3 Asahimachi, Abeno-ku, Osaka 545-8585, Japan
}

\author{
Eiko Yabuuchi, ${ }^{1}$ Yoshimasa Kosako, ${ }^{2}$ Nagatoshi Fujiwara, ${ }^{3}$ \\ Takashi Naka, ${ }^{3,4}$ Isamu Matsunaga, ${ }^{5}$ Hisashi Ogura ${ }^{5}$ and \\ Kazuo Kobayashi ${ }^{3}$

\begin{abstract}
Author for correspondence: Kazuo Kobayashi. Tel: +8166645 3745. Fax: +81666463662
\end{abstract} \\ e-mail:kobayak@med.osaka-cu.ac.jp
}

\begin{abstract}
The 165 rDNA sequence similarities between the type strains of Sphingomonas paucimobilis and 32 other Sphingomonas species range from $90 \cdot 2$ to $99.6 \%$. It might be possible to divide the genus into several new genera according to a dendrogram drawn from 165 rDNA sequence similarity. However, the phenotypic and biochemical information needed to define clusters of strains representing distinct genera within this group of organisms was not previously available. Although the cellular lipids of type strains of all 28 Sphingomonas species tested contained glucuronosyl-(1 $\rightarrow 1)$-ceramide together with 2-hydroxymyristic acid, other molecular species of sphingoglycolipids were distributed randomly. Sphingomonas natatoria and Sphingomonas ursincola, bacteriochlorophyll a-containing, Gram-negative facultative phototrophs, belong to the cluster of the genus Sphingomonas. Other phototrophic Porphyrobacter and Erythrobacter species in the Sphingomonadaceae were classified into a cluster different from the genus Sphingomonas, as reported previously. None of the physiological and biochemical characteristics considered, including cellular lipids and fatty acid composition, provided evidence for the division of the current genus Sphingomonas. It is therefore concluded that the genus Sphingomonas should remain undivided at this time. The species of three recently proposed genera, Sphingobium, Novosphingobium and Sphingopyxis, in conjunction with Blastobacter ursincola, are junior objective synonyms of species of the genus Sphingomonas.
\end{abstract}

Keywords: glucuronosyl ceramide, 2-hydroxymyristic acid, bacteriochlorophyll $a$, alcapton

\section{INTRODUCTION}

Delineation of the genus Sphingomonas has been the subject of taxonomic discussion by various workers (Balkwill et al., 1997; Hiraishi et al., 2000; Kämpfer et al., 1997; Stolz et al., 2000; Takeuchi et al., 1994,

Abbreviation: SGL, sphingoglycolipid.
2001). The recent detection of bacteriochlorophyll $a$ from cells of Blastomonas natatoria DSM $3183^{\mathrm{T}}$ and puf genes encoding proteins of the photosynthetic reactions detected by PCR (Hiraishi et al., 2000) has raised new interest. Based on the results of DNADNA hybridization and phylogenetic analysis of $16 \mathrm{~S}$ rDNA sequences of $B$. natatoria DSM $3183^{\mathrm{T}}$ and Erythromonas ursincola DSM $9006^{\mathrm{T}}$, the latter species has been transferred to the genus Blastomonas in a 
Table 1. History and growth conditions of type strains of 33 Sphingomonas species

Authorities are given for each name; these papers are not included in the reference list unless cited elsewhere.

\begin{tabular}{|c|c|c|c|}
\hline Strain & Received as & Source of isolation & $\begin{array}{l}\text { Medium and incubation } \\
\text { temperature }\left({ }^{\circ} \mathrm{C}\right)^{*}\end{array}$ \\
\hline Sphingomonas adhaesiva Yabuuchi et al. $1990 \mathrm{EY} 4215^{\mathrm{T}}$ & GIFU $11458^{\mathrm{T}}$ & 'Sterile' water used before surgery & Heart infusion agar, 30 \\
\hline Sphingomonas alaskensis Vancanneyt et al. $2001 \mathrm{EY} 4374^{\mathrm{T}}$ & DSM $13593^{\mathrm{T}}$ & Sea-water sample from Gulf of Alaska & Tryptic soy agar, 30 \\
\hline Sphingomonas aquatilis Lee et al. $2001 \mathrm{KCTC} 2881^{\mathrm{T}}$ & КCTC $2881^{\mathrm{T}}$ & Natural mineral water, Taejon, Korea & \\
\hline Sphingomonas aromaticivorans Balkwill et al. $1997 \mathrm{EY} 4296^{\mathrm{T}}$ & IFO $16084^{\mathrm{T}}$ & Terrestrial subsurface sediments & Tryptic soy agar, 30 \\
\hline Sphingomonas asaccharolytica Takeuchi et al. $1995 \mathrm{EY} 4229^{\mathrm{T}}$ & IFO $15499^{\mathrm{T}}$ & Roots of Malus spp. & PYA, 26 \\
\hline $\begin{array}{l}\text { Sphingomonas capsulata (Leifson 1962) } \\
\text { Yabuuchi et al. } 1990 \mathrm{EY} 4216^{\mathrm{T}}\end{array}$ & GIFU $11526^{\mathrm{T}}$ & Distilled water & Heart infusion agar, 26 \\
\hline Sphingomonas chlorophenolica Nohynek et al. $1996 \mathrm{EY} 4219^{\mathrm{T}}$ & ATCC $33790^{\mathrm{T}}$ & Pentachlorophenol-contaminated soil & $1 / 2$ TSA \\
\hline Sphingomonas chungbukensis Kim et al. $2000 \mathrm{EY} 4375^{\mathrm{T}}$ & JCM $11454^{\mathrm{T}}$ & Freshwater sediment, Taejon, Korea & Difco nutrient agar, 30 \\
\hline Sphingomonas cloacae Fujii et al. 2001 EY 4361 & JCM $10874^{\mathrm{T}}$ & Sewage-treatment-plant water sample, Tokyo & Heart infusion agar, 30 \\
\hline $\begin{array}{l}\text { Sphingomonas echinoides (Heumann 1962) } \\
\text { Denner } \text { et al. } 1999 \mathrm{EY} 4340^{\mathrm{T}}\end{array}$ & JCM $10637^{\mathrm{T}}$ & Plate contaminant & $1 / 2$ TSA, 30 \\
\hline Sphingomonas herbicidovorans Zipper et al. 1997 EY 4344 ${ }^{\mathrm{T}}$ & DSM $11019^{\mathrm{T}}$ & Soil & Tryptic soy agar, 28 \\
\hline Sphingomonas koreensis Lee et al. $2001 \mathrm{EY} 4376^{\mathrm{T}}$ & $\mathrm{JCM} 11456^{\mathrm{T}}$ & Natural mineral water, Taejon, Korea & Difco nutrient agar, 30 \\
\hline Sphingomonas macrogoltabidus Takeuchi et al. $1993 \mathrm{EY} 4304^{\mathrm{T}}$ & JCM $10192^{\mathrm{T}}$ & Soil & Tryptic soy agar, 30 \\
\hline Sphingomonas mali Takeuchi et al. $1995 \mathrm{EY} 4341^{\mathrm{T}}$ & IFO $15500^{\mathrm{T}}$ & Roots of Malus spp. & $1 / 2$ TSA, 30 \\
\hline Sphingomonas natatoria (Sly 1985) & ATCC $35951^{\mathrm{T}}$ & Freshwater swimming pool & PYA, 26 \\
\hline Yabuuchi et al. $1999 \mathrm{EY} 4220^{\mathrm{T}}$ & & & \\
\hline Sphingomonas parapaucimobilis Yabuuchi et al. $1990 \mathrm{EY} 4213^{\mathrm{T}}$ & GIFU $11387^{\mathrm{T}}$ & Urine & Heart infusion agar, 30 \\
\hline $\begin{array}{l}\text { Sphingomonas paucimobilis (Holmes et al. 1977) } \\
\text { Yabuuchi et al. } 1990 \text { EY } 2395^{\mathrm{T}}\end{array}$ & GIFU $2395^{\mathrm{T}}$ & Hospital mechanical respirator & Heart infusion agar, 30 \\
\hline Sphingomonas pituitosa Denner et al. $2001 \mathrm{EY} 4370^{\mathrm{T}}$ & DSM $13101^{\mathrm{T}}$ & Eutrophic fountain water, Vienna, Austria & Tryptic soy agar, 30 \\
\hline Sphingomonas pruni Takeuchi et al. 1995 EY $4228^{\mathrm{T}}$ & IFO $15498^{\mathrm{T}}$ & Roots of Prunus persica & PYA, 26 \\
\hline Sphingomonas rosa Takeuchi et al. 1995 EY $4227^{\mathrm{T}}$ & IFO $15208^{\mathrm{T}}$ & Hairy roots of Rosa spp. & PYA, 26 \\
\hline Sphingomonas roseiflava Yun et al. $2000 \mathrm{EY} 4345^{\mathrm{T}}$ & JCM $99038^{\mathrm{T}}$ & Plant (Setaria viridis) & Tryptic soy agar, 28 \\
\hline Sphingomonas sanguinis Takeuchi et al. $1993 \mathrm{EY} 2397^{\mathrm{T}}$ & GIFU $2397^{\mathrm{T}}$ & Blood & Heart infusion agar, 30 \\
\hline Sphingomonas stygia Balkwill et al. $1997 \mathrm{EY} 4297^{\mathrm{T}}$ & IFO $16085^{\mathrm{T}}$ & As S. aromaticivorans IFO $16084^{\mathrm{T}}$ & Tryptic soy agar, 30 \\
\hline Sphingomonas subarctica Nohynek et al. $1996 \mathrm{EY} 4251^{\mathrm{T}}$ & DSM $10700^{\mathrm{T}}$ & Biofilm of reactor inoculated with activated sludge & Tryptic soy agar, 28 \\
\hline $\begin{array}{l}\text { Sphingomonas suberifaciens (van Burgen et al. 1990) } \\
\text { Yabuuchi et al. } 1999 \mathrm{EY} 4204^{\mathrm{T}}\end{array}$ & JCM $8521^{\mathrm{T}}$ & Corky root of lettuce & ATCC medium 1700, 26 \\
\hline Sphingomonas subterranea Balkwill et al. $1997 \mathrm{EY} 4298^{\mathrm{T}}$ & IFO $16086^{\mathrm{T}}$ & As S. aromaticivorans IFO $16084^{\mathrm{T}}$ & Tryptic soy agar, 30 \\
\hline Sphingomonas taejonensis Lee et al. $2001 \mathrm{EY} 4377^{\mathrm{T}}$ & $\mathrm{JCM} 11457^{\mathrm{T}}$ & Natural mineral water, Taejon, Korea & Difco nutrient agar \\
\hline Sphingomonas terrae Takeuchi et al. $1993 \mathrm{EY} 4207^{\mathrm{T}}$ & IFO $15098^{\mathrm{T}}$ & Activated sludge & Tryptic soy agar \\
\hline Sphingomonas trueperi Kämpfer et al. $1997 \mathrm{EY} 4218^{\mathrm{T}}$ & ATCC $12417^{\mathrm{T}}$ & Soil & Tryptic soy agar,26 \\
\hline $\begin{array}{l}\text { Sphingomonas ursincola (Yurkov et al. 1997) } \\
\text { Yabuuchi et al. } 1999 \mathrm{EY} 4250^{\mathrm{T}}\end{array}$ & DSM $9006^{\mathrm{T}}$ & Freshwater cyanobacterial mat & $\mathrm{NA}, 26$ \\
\hline Sphingomonas wittichii Yabuuchi et al. $2001 \mathrm{EY} 4224^{\mathrm{T}}$ & DSM $6014^{\mathrm{T}}$ & Water from the River Elbe & TSA, 30 \\
\hline Sphingomonas xenophaga Stolz et al. $2000 \mathrm{EY} 4343^{\mathrm{T}}$ & DSM $6383^{\mathrm{T}}$ & Water from the River Elbe & Tryptic soy agar, 28 \\
\hline Sphingomonas yanoikuyae Yabuuchi et al. $1990 \mathrm{EY} 4208^{\mathrm{T}}$ & GIFU $9882^{\mathrm{T}}$ & Clinical specimen & Tryptic soy agar, 28 \\
\hline
\end{tabular}

* Heart infusion agar and tryptic soy agar were from Difco. ATCC medium 1700 is Rhizobium medium. Other media are abbreviated as NA (0.5 g Bacto peptone, $0 \cdot 3 \mathrm{~g}$ Bacto yeast extract and $1.5 \mathrm{~g}$ agar in $100 \mathrm{ml}), 1 / 2 \mathrm{NA}(0.25 \mathrm{~g}$ Bacto peptone, $0.15 \mathrm{~g}$ Bacto yeast extract and $1.5 \mathrm{~g}$ agar in $100 \mathrm{ml})$, PYA (1.0 g Bacto peptone, $0.2 \mathrm{~g}$ Bacto yeast extract, $0.2 \mathrm{~g} \mathrm{NaCl}$ and $1.2 \mathrm{~g}$ agar in $100 \mathrm{ml})$ and $1 / 2$ TSA $(2 \cdot 0 \mathrm{~g}$ Bacto tryptic soy broth and $1.5 \mathrm{~g}$ agar in $100 \mathrm{ml})$. 
proposed new combination, Blastomonas ursincola (Yurkov et al. 1997) Hiraishi et al. 2000.

However, it had been proposed previously that $B$. natatoria DSM $3183^{\mathrm{T}}$ and Erythromonas ursincola DSM $9006^{\mathrm{T}}$ belong to the genus Sphingomonas, as Sphingomonas natatoria (Sly 1985) Yabuuchi et al. 1999 and Sphingomonas ursincola (Yurkov et al. 1997) Yabuuchi et al. 1999, by the study of polyphasic taxonomy (Yabuuchi et al., 1999). The presence of bacteriochlorophyll $a$ and facultative phototrophic ability are unlikely to indicate a close phylogenetic relationship in prokaryotes (Kondratieva et al., 1992). Other phototrophic organisms, such as Erythrobacter longus, Erythrobacter litoralis, Erythromicrobium ramosum and Porphyrobacter tepidarius (Hanada et al., 1997), are outside the genus Sphingomonas, but included in Sphingomonadaceae Kosako et al. 2000, according to the presence of glucuronosyl ceramide in their cellular lipids and phylogenetic analysis of $16 \mathrm{~S}$ rDNA nucleotide sequences (Kosako et al., 2000).

On the basis of phylogenetic analysis of 16S rDNA sequences and polyamine profiles, Takeuchi et al. (2001) proposed that the genus Sphingomonas be classified into four genera, Sphingomonas, Sphingobium, Novosphingobium and Sphingopyxis. From a practical aspect, it is essential that any genus should not be delineated by phylogenetic analysis alone, but should also be distinguished by a combination of phenotypic tests that are performed easily in every microbiological laboratory. In this paper, we have examined the phenotypic characteristics of species currently assigned to the genus Sphingomonas (Yabuuchi et al. 1990) in relation to their phylogeny and found that there is no phenotypic evidence to support a proposal to split the species into four genera. We thus conclude that the genus Sphingomonas should remain undivided at this time.

\section{METHODS}

Bacterial strains. The history and growth conditions of the type strains of 33 Sphingomonas species are listed in Table 1. Two of them were isolated from clinical specimens, two from a hospital environment, one from a diseased plant and the remaining 28 were of environmental origin. Growth media and incubation temperatures for each type strain are also listed in Table 1.

16S rDNA sequences and phylogenetic analysis. Deposited data on $16 \mathrm{~S}$ rDNA sequences of the type strains of 33 Sphingomonas species, two subspecies of Zymomonas mobilis as a representative member of Sphingomonadaceae and Acetobacter aceti as an outgroup organism were obtained from the DNA Database of Japan (DDBJ, Institute of Genetics, Shizuoka, Japan) and were used for phylogenetic analysis. Sequence accession numbers of bacterial strains used in this study are shown in Fig. 1. Phylogenetic analysis by $16 \mathrm{~S}$ rDNA oligonucleotide sequences was performed as described previously (Kosako et al., 2000). In brief, nucleotide substitution rates $\left(K_{\text {nuc }}\right.$; Kimura, 1980) were determined and a distance-matrix tree was constructed by the neighbourjoining method (Saitou \& Nei, 1987) using the program CLUSTAL w (Thompson et al., 1994). Alignment gaps and unidentified base positions were excluded from the calculation. To evaluate the topology of the phylogenetic tree, a bootstrap analysis was performed (Yabuuchi et al., 1999; Kosako et al., 2000).

Phenotypic characterization and carbon assimilation. Phenotypic features were determined by the methods of Yabuuchi et al. $(1990,1999)$. Production of oxidative acids from sugars and polyalcohols was determined using OF basal medium (Difco), except for three strains, Sphingomonas xenophaga EY 4343 ${ }^{\mathrm{T}}$, Sphingomonas herbicidovorans EY $4344^{\mathrm{T}}$ and Sphingomonas roseiflava EY $4345^{\mathrm{T}}$, that were unable to grow in OF basal medium. To analyse these strains, cystine tryptic agar (Difco) was used. Assimilation tests were performed by using Biotype 100 and Biotype medium (bioMérieux) in accordance with the manufacturer's instructions. API 20NE (bioMérieux) was also used for characterization of organisms.

Production of homogentisic acid/alcapton/2,5-dihydroxyphenylacetic acid. Culture supernatants of all bacterial strains used in the present study, including Sphingomonas herbicidovorans EY $4344^{\mathrm{T}}$ and Pseudomonas aeruginosa ATCC 23268, standard producers of homogentisic acid (Yabuuchi \& Ohyama, 1972), were tested for production of homogentisic acid during the metabolism of phenylalanine and tyrosine by using HPLC (Bory et al., 1990; Mathieu et al., 1997).

Antimicrobial susceptibility testing. Except for Sphingomonas suberifaciens EY 4204 ${ }^{\mathrm{T}}$, Sphingomonas pruni EY 4228 ${ }^{\mathrm{T}}$, Sphingomonas asaccharolytica EY 4229 ${ }^{\mathrm{T}}$ and Sphingomonas mali EY 4341 ${ }^{\mathrm{T}}$, antimicrobial susceptibility testing was performed using Sensi-discs and Mueller-Hinton II plates (Difco) (Kosako et al., 2000; Yabuuchi et al., 1999, 2001). Sphingomonas suberifaciens EY $4204^{\mathrm{T}}$ was tested using ATCC medium 1700 supplemented with 1.5\% agar (Yabuuchi et al., 1999). Peptone/yeast extract agar was used for growth of Sphingomonas pruni EY $4228^{\mathrm{T}}$ and Sphingomonas asaccharolytica EY $4229^{\mathrm{T}}$ and a $50 \%$ concentration of nutrient broth supplemented with $1.5 \%$ agar was used for Sphingomonas mali EY $4341^{\mathrm{T}}$.

Analysis of cellular lipids and fatty acids. Bacterial cells grown on agar media at optimal temperatures were harvested to analyse cellular lipids and fatty acids as described previously (Naka et al., 2000; Yabuuchi et al., 1990, 1999).

\section{RESULTS AND DISCUSSION}

\section{Phylogenetic analysis}

Fig. 1 shows a dendrogram derived from 16S rDNA oligonucleotide sequence similarity among the type strains of 33 Sphingomonas species and Acetobacter aceti as an outgroup micro-organism. From Sphingomonas parapaucimobilis to Sphingomonas wittichii, including Sphingomonas paucimobilis, the type species of the genus, it seems to form a single large branch. From Sphingomonas chlorophenolica to Sphingomonas terrae, 17 species are divided into three branches. The type strain of Sphingomonas suberifaciens belongs to the branch of Sphingomonas chlorophenolica. Both Sphingomonas ursincola and Sphingomonas natatoria are included in the Sphingomonas chlorophenolicaSphingomonas terrae branch. Numerical analysis of 27 type strains of Sphingomonas species demonstrates that the members of three new genera proposed by 


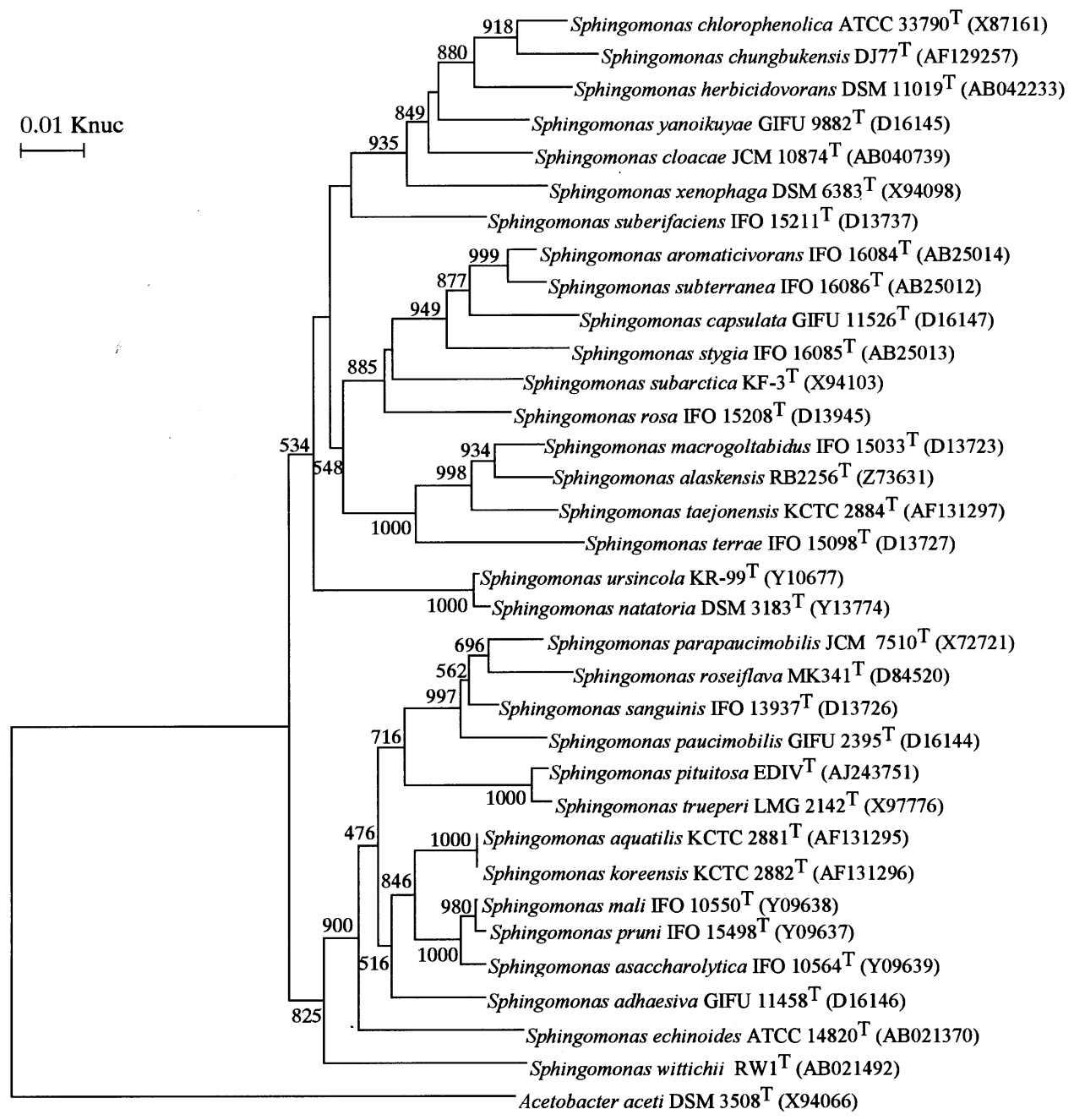

Fig. 1. Dendrogram based on phylogenetic analysis of $16 \mathrm{~S}$ rDNA sequence of type strains of 33 Sphingomonas species. Nucleotide substitution rates $\left(K_{\text {nuc }}\right.$ ) (Kimura, 1980) were determined using the program CLUSTAL W (Thompson et al., 1994). The neighbour-joining method (Saitou \& Nei, 1987) was used to reconstruct a phylogenetic tree from the distance matrices by using NJPLOT. Alignment gaps and unidentified base positions were excluded from the calculation and final analysis. To evaluate the topology of the phylogenetic tree, a bootstrap analysis was performed using 1000 bootstrapped trials. Bootstrap values are indicated at branching points.

Takeuchi et al. (2001) do not form solid clusters (Fig. 2). Our results do not support the proposal of the genus Sphingomonas and three new genera, Sphingobium, Novosphingobium and Sphingopyxis, on the basis of phylogenetic and chemotaxonomic analyses.

\section{Phenotypic characteristics}

Colonies of Sphingomonas natatoria EY $4220^{\mathrm{T}}$ and Sphingomonas ursincola EY $4250^{\mathrm{T}}$, facultatively phototrophic organisms, were orange-coloured. At harvest, however, they gave a melted-chocolate-like colour. Sphingomonas herbicidovorans EY $4344^{\mathrm{T}}$ produced water-soluble melanin-like pigment on heart infusion or tryptic soy agar plates. Grown slowly on Bactophenylalanine agar slants, Sphingomonas herbicidovorans EY $4344^{\mathrm{T}}$ turned the medium pink from the surface. The culture supernatant of Sphingomonas herbicidovorans EY $4344^{\mathrm{T}}$, but not other strains, contained alcapton/homogentisic acid (Mann, 1979). Because of its nutritional requirements, both API 20NE and Biotype 100 enabled us to estimate the assimilating ability of Sphingomonas suberifaciens EY $4204^{\mathrm{T}}$. A combination of certain phenotypic features was useful to differentiate strains at the species level, but not groups of strains at the generic level (Tables 2-4 and Fig. 2).

\section{Distribution of sphingoglycolipids (SGLs) and major polyamines}

Glucuronosyl-(1 $\rightarrow 1$ )-ceramide (SGL-1), but not galacturonosyl-( $1 \rightarrow 1)$-ceramide (SGL-1'), was present in cellular lipids of all 28 strains tested (Table 5). Other SGLs (SGL-2, -3 and -4) and fatty acids, such as 2-OH 15:0 and 2-OH 16:0, could not contribute to the 


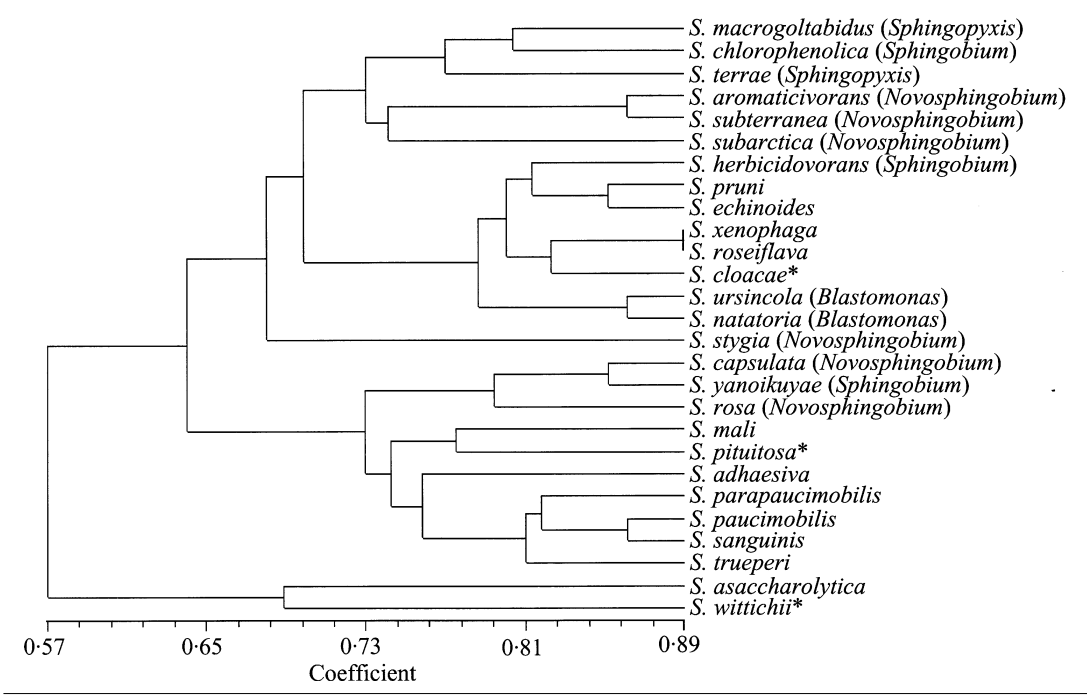

Fig. 2. Numerical analysis of 27 type strains of Sphingomonas species. Numerical analysis was performed by using NTSYS-pc version 2 (Applied Biostatistics). The coefficient of simple matching was used for calculation and the dendrogram was depicted by UPGMA (Sneath \& Sokal, 1973). S., Sphingomonas. Genera in parentheses follow Takeuchi et al. (2001). *, Not included in Takeuchi et al. (2001).

division of the genus. Major polyamines detected were identified as spermidine and homospermidine (Hamana \& Matsuzaki, 1993; Segers et al., 1994; Stolz et al., 2000; Takeuchi et al., 1995).

\section{Antimicrobial susceptibility of Sphingomonas species}

All strains tested were susceptible (S) or showed an intermediate response (IM) to antimicrobials such as tetracycline, doxycycline and amikacin (Table 6). Meropenem, panipenem, amoxicillin/clavulanic acid, minocycline and imipenem exerted antimicrobial activity against most strains used in this study. Sphingomonas suberifaciens EY 4204 ${ }^{\mathrm{T}}$, Sphingomonas natatoria EY $4220^{\mathrm{T}}$, Sphingomonas roseiflava EY $4345^{\mathrm{T}}$ and Sphingomonas paucimobilis EY $2395^{\mathrm{T}}$ were each susceptible to more than 30 of 36 discs. Although isolates from the clinical specimen and from the hospital environment were susceptible to fluoroquinolones, 12 strains originating from plants, soil and other sources were resistant to certain or all new fluoroquinolones tested. The type strains of Sphingomonas pruni and Sphingomonas rosa were resistant to more than $50 \%$ of discs tested. Again, these results indicate that the pattern of antimicrobial susceptibility/resistance does not provide any convenient basis for subdividing the genus.

Phototrophic ability is distributed widely in prokaryotes and does not necessarily indicate a close phylogenetic relationship (Kondratieva et al., 1992). Sphingomonas natatoria and Sphingomonas ursincola are closely related phylogenetically within the genus Sphingomonas (Yabuuchi et al., 1999). Both Sphingomonas ursincola and Sphingomonas natatoria have been identified as phototrophs by HPLC analysis of photosynthetic pigments and genetic studies (Hiraishi et al., 2000). In addition, flagella morphology is unlikely to be a key feature to distinguish a genus from others (Yabuuchi et al., 1995). The presence of bacteriochlorophyll $a$ is not a reason, in isolation, to exclude two phylogenetically related and facultatively phototrophic species, Sphingomonas natatoria and Sphingomonas ursincola, from the genus Sphingomonas.

The division of the genus Sphingomonas into four genera has been proposed on the basis of sequence analysis of $16 \mathrm{~S}$ rDNA, including signature sequences, and polyamine patterns (Busse \& Auling, 1988; Hamana \& Matsuzaki, 1993; Takeuchi et al., 1995). More recently, similar methods were used to propose three new genera derived from the genus Sphingomonas (Takeuchi et al., 2001). In the present study, we have examined 28 type strains of Sphingomonas species by phenotypic, biochemical and genetic analyses. We have shown that there is no phenotypic and phylogenetic evidence to support a proposal to divide the species into new genera. Therefore, we have concluded that the genus Sphingomonas should remain undivided at this time. The species of three recently proposed genera, Sphingobium, Novosphingobium and Sphingopyxis, in conjunction with Blastobacter ursincola, are junior objective synonyms of species of the genus Sphingomonas.

\section{Emended description of the genus Sphingomonas Yabuuchi et al. 1990}

Gram-negative, asporogenous rods or ovoid cells. Soma size is $0.7 \times 1.4 \mu \mathrm{m}, 0.4 \times 0.9 \mu \mathrm{m}$ (Sphingomonas suberifaciens) or $0.9 \times 2.0 \mu \mathrm{m}$ (Sphingomonas ursincola). Budding or asymmetric division is visualized by electron microscopy in two species (Sphingomonas natatoria and Sphingomonas ursincola). Bipolar staining in Gram stain (Sphingomonas ursincola) and rosette formation in some species. Usually single polar flagellum when motile. Sphingomonas trueperi is peritrichous. Sphingomonas chlorophenolica, Sphingomonas cloacae, Sphingomonas xenophaga, Sphingomonas aromaticivorans, Sphingomonas subterranea, Sphingomonas capsulata, Sphingomonas stygia, Sphingomonas subarctica, Sphingomonas rosa, 
Table 2. Physiological and biological characteristics of 28 type strains of Sphingomonas species

Strains are arranged in the order of the dendrogram in Fig. 1. Sphingomonas paucimobilis is the type species of the genus. Strains (S., Sphingomonas): 1, S. chlorophenolica

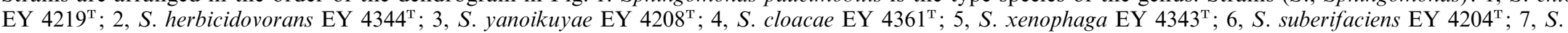

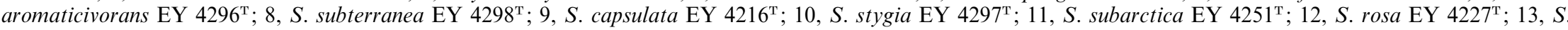

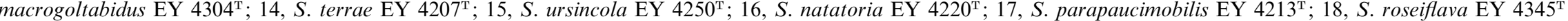

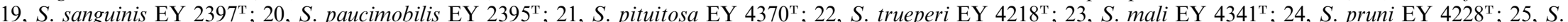

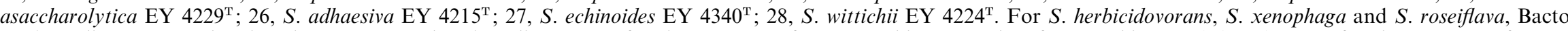
CTA medium was used rather than Bacto OF basal medium. Data for the presence of nostoxanthin were taken from Jenkins et al. (1979). Data for the presence of bacteriochlorophyll $a$ were taken from Yurkov et al. (1997) and Hiraishi et al. (2000). Oxidative acid production was tested using Bacto OF basal medium. Characters are scored as: + , positive reaction within 3 days; $(+)$, positive reaction after more than 4 days; - , negative reaction; ND, no data; NG, no growth.

\begin{tabular}{|c|c|c|c|c|c|c|c|c|c|c|c|c|c|c|c|c|c|c|c|c|c|c|c|c|c|c|c|c|}
\hline Characteristic & 1 & 2 & 3 & 4 & 5 & 6 & 7 & 8 & 9 & 10 & 11 & 12 & 13 & 14 & 15 & 16 & 17 & 18 & 19 & 20 & 21 & 22 & 23 & 24 & 25 & 26 & 27 & 28 \\
\hline Colony colour* & - & $\mathrm{Y}$ & (Y) & $\mathrm{GW}$ & $\mathrm{Y}$ & - & $\mathrm{Y}$ & $\mathrm{Y}$ & $\mathrm{Y}$ & $\mathrm{Y}$ & - & - & $(\mathrm{Y})$ & $(\mathrm{Y})$ & o & o & $\mathrm{Y}$ & PY & $\mathrm{Y}$ & $\mathrm{Y}$ & $\mathrm{Y}$ & $\mathrm{Y}$ & LY & $\mathrm{Y}$ & $\mathrm{Y}$ & $\mathrm{Y}$ & $\mathrm{Y}$ & (Y) \\
\hline Nostoxanthin & $\mathrm{ND}$ & ND & ND & ND & ND & ND & ND & ND & ND & ND & $\mathrm{ND}$ & ND & ND & ND & ND & ND & ND & ND & + & + & $\mathrm{ND}$ & + & ND & ND & $\mathrm{ND}$ & $\mathrm{ND}$ & + & ND \\
\hline Motility & - & + & + & - & - & + & - & - & - & - & - & - & + & + & - & - & + & - & + & + & + & + & + & - & - & + & + & + \\
\hline Flagellation $\dagger$ & & ND & $1 \mathrm{P}$ & & & $1 \mathrm{P}$ & & & & & & & $1 \mathrm{P}$ & $1 \mathrm{P}$ & & $1 \mathrm{P}$ & $1 \mathrm{P}$ & & $1 \mathrm{P}$ & $1 \mathrm{P}$ & $1 \mathrm{P}$ & Peri & ND & & & $1 \mathrm{P}$ & $1 \mathrm{P}$ & $1 \mathrm{P}$ \\
\hline Oxidase (Kovacs) & + & - & - & - & + & + & + & + & - & + & + & - & + & + & + & - & - & - & - & - & + & - & + & + & + & - & - & + \\
\hline $\begin{array}{l}\text { Bacteriochlorophyll } a \\
\text { Hydrolvsis of: }\end{array}$ & - & - & - & - & - & - & - & - & - & - & - & - & - & - & + & + & - & - & - & - & - & - & - & - & - & - & - & - \\
\hline Aesculin & + & + & + & + & + & + & + & + & + & + & + & + & + & + & $(+)$ & + & + & + & + & + & + & + & + & + & + & + & + & + \\
\hline Gelatin & + & + & - & - & - & - & + & + & - & + & + & - & + & + & + & - & - & - & + & + & - & - & - & - & - & + & + & + \\
\hline Starch & - & - & - & - & - & - & + & - & + & - & - & - & + & - & + & + & + & + & + & + & + & - & - & $(+)$ & + & + & + & - \\
\hline Tween 80 & - & - & - & - & + & - & - & - & + & - & - & - & + & - & + & + & + & + & + & + & + & - & + & + & + & + & - & - \\
\hline Citrate (Simmons) & + & - & + & - & - & - & - & - & - & - & + & - & + & $(+)$ & - & - & - & - & - & + & - & - & - & - & - & - & + & - \\
\hline DNase & + & - & - & - & - & - & + & + & + & + & + & + & - & + & - & $(+)$ & - & - & - & - & - & $(+)$ & + & NG & NG & - & - & - \\
\hline Phenylalanine deaminase & - & - & - & - & + & - & - & - & + & - & - & - & - & - & - & - & - & - & - & + & - & - & - & - & - & - & - & - \\
\hline Acylamidase & - & - & - & - & - & - & - & - & - & - & $(+)$ & - & - & - & - & - & - & - & - & - & - & - & - & NG & NG & - & + & + \\
\hline $\begin{array}{l}\text { Alcapton production } \\
\text { Oxidative acid from: }\end{array}$ & - & + & - & - & - & - & - & - & - & - & - & - & - & - & - & - & - & - & - & - & - & - & - & - & - & - & - & - \\
\hline Glucose & + & + & + & - & + & NG & + & + & + & + & + & $(+)$ & + & - & $(+)$ & - & - & + & + & + & + & + & + & - & $(+)$ & + & + & + \\
\hline Maltose & - & + & + & $(+)$ & + & NG & + & + & + & + & + & + & + & - & + & + & - & + & + & + & - & + & + & + & + & + & + & + \\
\hline Sucrose & + & - & + & - & $(+)$ & NG & + & + & + & + & + & + & + & - & + & + & + & + & + & + & + & + & - & - & - & + & + & + \\
\hline D-Ribose & - & - & + & - & - & NG & + & + & + & - & + & $(+)$ & $(+)$ & + & + & + & - & - & - & - & - & - & - & - & - & - & - & + \\
\hline Xylose & + & + & + & - & + & NG & + & + & + & + & - & - & + & - & - & - & $(+)$ & + & + & + & - & + & + & + & $(+)$ & + & + & + \\
\hline Dulcitol & + & $(+)$ & - & - & + & NG & + & + & - & NG & $(+)$ & NG & - & + & + & + & - & - & - & - & - & $(+)$ & + & - & + & - & - & - \\
\hline Sorbitol & - & - & - & - & - & NG & - & - & - & + & $(+)$ & - & + & + & - & - & - & - & - & - & - & - & - & $(+)$ & - & - & + & + \\
\hline Mannitol & + & $(+)$ & - & - & - & NG & + & + & - & + & + & - & - & + & + & + & - & - & - & - & - & $(+)$ & - & - & + & - & - & + \\
\hline Inositol & - & - & - & - & - & NG & - & - & - & + & - & - & - & - & - & + & - & - & - & - & $(+)$ & - & + & - & - & - & - & - \\
\hline Adonitol & + & $(+)$ & - & - & - & NG & + & - & - & - & - & - & - & - & - & + & - & - & - & - & - & - & - & - & - & - & - & - \\
\hline
\end{tabular}

*y, Deep yellow; o, orange; PY, pink-yellow; LY, lemon-yellow; (Y), yellowish after 3 or more days at room temperature.

$\dagger 1 \mathrm{P}$, Polar mono; Peri, peritrichous. 
Table 3. Selected features of the assimilation profiles of type strains of 28 Sphingomonas species obtained by Biotype 100

See Table 2 for identities of strains. +, Positive growth by substrate assimilation; - , substrate not assimilated. Final reading was performed after 7 days cultivation at $30{ }^{\circ} \mathrm{C}$. The following compounds were not assimilated by any of the 28 type strains tested: dulcitol, $\mathrm{L}(+)$-sorbose, $\mathrm{D}(+)$-arabitol, $\mathrm{L}(-)$-arabitol, D-sorbitol, adonitol, hydroxyquinoline $\beta$-glucuronide, $\mathrm{L}(+)$-tartrate, 3-phenylpropionate, $m$-coumarate, trigonelline, histamine, DL- $\alpha$-amino- $n$-valerate, histidine, ethanolamine, malonate, 3 - $o$ methyl D-glucopyranose, tryptophan.

\begin{tabular}{|c|c|c|c|c|c|c|c|c|c|c|c|c|c|c|c|c|c|c|c|c|c|c|c|c|c|c|c|c|}
\hline Substrate & 1 & 2 & 3 & 4 & 5 & 6 & 7 & 8 & 9 & 10 & 11 & 12 & 13 & 14 & 15 & 16 & 17 & 18 & 19 & 20 & 21 & 22 & 23 & 24 & 25 & 26 & 27 & 28 \\
\hline D-Galacturonate & - & - & - & - & - & - & + & - & - & - & - & - & - & - & - & - & - & - & - & - & - & + & - & + & - & - & - & - \\
\hline Phenylacetate & - & - & - & - & - & - & - & + & - & - & - & - & - & - & - & - & - & - & - & - & - & - & - & - & + & - & - & + \\
\hline 2-Oxoglutarate & - & - & - & - & - & - & - & - & - & + & - & - & - & - & - & - & + & - & - & - & + & - & - & - & - & + & - & - \\
\hline 2-Keto-D-gluconate & - & - & - & - & - & - & - & - & - & + & - & - & - & - & - & - & + & - & - & - & - & - & - & - & - & - & - & - \\
\hline Benzoate & - & - & - & - & - & - & - & - & - & + & + & + & - & - & - & - & - & - & - & - & - & - & - & - & - & - & - & - \\
\hline $\mathrm{D}(-)$-Tartrate & - & - & - & - & - & - & - & - & - & - & - & - & + & + & - & - & - & - & - & - & + & - & - & - & - & - & - & - \\
\hline D-Mannitol & - & - & - & - & - & - & - & - & - & - & - & - & - & - & + & - & - & - & - & - & - & - & - & - & + & - & - & + \\
\hline Citrate & - & - & + & - & - & - & - & - & - & - & - & - & - & - & - & - & + & - & - & + & - & - & - & - & + & - & - & + \\
\hline L-Alanine & + & - & - & - & - & - & + & + & - & - & + & - & - & + & - & + & - & - & - & - & + & - & - & + & + & - & - & - \\
\hline $\mathrm{D}(+)$-Xylose & - & - & + & + & + & - & + & + & + & - & + & + & - & - & - & + & + & + & + & + & + & + & + & + & - & - & - & + \\
\hline L(-)-Malate & + & + & + & - & + & - & - & - & + & - & + & - & - & + & - & - & + & + & - & + & + & + & & + & + & + & + & + \\
\hline L-Glutamate & + & - & + & + & - & - & + & + & + & + & + & - & + & + & - & + & + & - & + & + & + & + & + & + & + & + & - & + \\
\hline$\alpha$-L-Rhamnose & - & + & + & - & - & - & + & + & + & - & + & + & - & - & - & - & + & - & - & - & - & - & - & - & - & - & - & - \\
\hline Succinate & - & - & + & - & + & - & - & - & + & + & + & + & - & - & - & - & + & + & + & + & + & + & - & - & + & + & - & + \\
\hline $\mathrm{D}(+)$-Mannose & - & - & + & - & - & - & + & + & + & - & - & + & - & - & + & - & + & - & + & + & + & + & - & + & + & + & + & + \\
\hline Lactulose & - & - & - & - & - & - & - & - & - & - & - & - & - & - & - & - & + & - & + & + & + & - & - & - & - & - & - & - \\
\hline D-Glucosamine & - & - & - & - & - & - & - & - & - & - & - & - & - & - & - & - & + & - & - & - & - & - & - & - & - & - & - & + \\
\hline Betaine & - & - & - & - & - & - & - & - & - & - & - & - & - & - & - & - & - & - & - & + & - & - & - & - & - & - & - & - \\
\hline Glutarate & - & - & - & - & - & - & - & - & - & - & - & - & - & - & - & - & - & - & - & + & - & - & - & - & + & - & - & - \\
\hline
\end{tabular}


Table 4. Results of API 20NE tests on type strains of 28 Sphingomonas species

See Table 2 for identities of strains. All 28 type strains were positive for the Zn-dust test and negative for the nitrite test, indole production from tryptophan, acid from glucose under mineral oil, arginine dihydrolase, urease and assimilation of D-mannitol. PNPG, $p$-Nitrophenyl $\beta$-D-galactopyranosidase.

\begin{tabular}{|c|c|c|c|c|c|c|c|c|c|c|c|c|c|c|c|c|c|c|c|c|c|c|c|c|c|c|c|c|}
\hline Substrate or test & 1 & 2 & 3 & 4 & 5 & 6 & 7 & 8 & 9 & 10 & 11 & 12 & 13 & 14 & 15 & 16 & 17 & 18 & 19 & 20 & 21 & 22 & 23 & 24 & 25 & 26 & 27 & 28 \\
\hline Aesculin & + & + & + & $*$ & + & + & + & + & + & + & + & + & + & + & + & + & + & + & + & + & + & + & + & + & + & + & + & $*$ \\
\hline PNPG & + & + & + & - & + & + & + & + & + & + & + & + & + & - & - & - & + & + & + & + & + & + & + & + & + & + & - & - \\
\hline \multicolumn{29}{|l|}{ Assimilation of: } \\
\hline Glucose & + & + & + & + & + & - & + & + & + & + & + & + & + & - & - & + & + & + & + & + & + & + & + & + & + & + & + & - \\
\hline L-Arabinose & - & - & + & + & + & - & - & + & + & + & + & + & - & - & - & + & + & + & + & + & + & + & + & + & + & & + & - \\
\hline D-Mannose & + & - & - & + & - & - & + & + & + & - & + & - & - & - & - & - & + & - & + & + & - & + & + & - & + & + & + & - \\
\hline$N$-Acetyl glucosamine & - & - & + & + & - & - & + & - & + & - & + & + & - & - & - & - & + & - & + & + & + & + & + & + & + & + & + & - \\
\hline Maltose & - & - & + & + & + & - & + & + & + & + & + & + & - & + & + & + & + & + & + & + & + & + & + & + & + & + & + & - \\
\hline Gluconate & - & - & + & + & - & - & - & - & + & - & - & - & - & - & - & - & + & + & + & + & + & - & + & - & - & - & + & - \\
\hline$n$-Capric acid & + & - & - & + & - & - & - & - & - & - & - & - & - & - & - & - & - & - & - & - & + & - & - & - & - & - & - & - \\
\hline Adipic acid & - & - & - & + & - & - & - & - & - & - & + & - & - & - & - & + & - & - & - & + & + & - & - & - & - & - & - & - \\
\hline DL-Malic acid & - & + & + & + & + & - & - & - & - & - & + & - & - & + & - & - & + & + & + & + & + & + & - & - & - & + & + & - \\
\hline Citrate & - & - & + & - & - & - & - & - & - & - & - & - & - & - & - & - & + & - & - & + & - & - & - & - & - & - & - & - \\
\hline Phenylacetate & - & - & - & - & - & - & - & - & - & - & - & - & - & - & - & - & - & - & - & - & - & - & - & - & - & - & - & + \\
\hline
\end{tabular}

* Negative on API 20NE, but positive on aesculin agar slants and Biotype 100 
Table 5. Selected cellular components characteristic for type strains of 28 Sphingomonas species

See Table 2 for identities of strains. All 28 type strains tested were positive for SGL-1 and ubiquinone 10. All 28 type strains tested were negative for 3-hydroxy fatty acids. tr, Less than $1 \% ;-$, not detected; ND, no data; CPA, cyclopropanoic acid.

\begin{tabular}{|c|c|c|c|c|c|c|c|c|c|c|c|c|c|c|c|c|c|c|c|c|c|c|c|c|c|c|c|c|c|}
\hline Cellular component & 1 & 2 & 3 & 4 & 5 & 6 & 7 & 8 & 9 & 10 & 11 & 12 & 13 & 14 & 15 & 16 & 17 & 18 & 19 & 20 & 21 & 22 & & & 24 & 25 & 26 & 27 & 2 \\
\hline \multicolumn{30}{|l|}{ Fatty acids $(\%)$ : } \\
\hline $16: 1 \omega 9 c$ & 7 & 5 & 14 & 3 & 22 & 13 & 16 & 11 & 2 & 18 & 6 & 17 & 29 & 4 & 9 & 5 & 3 & 13 & 3 & 2 & 1 & 1 & $\mathrm{t}$ & & ND & ND & 4 & 1 & 6 \\
\hline 17:0 CPA & $\operatorname{tr}$ & 1 & $\operatorname{tr}$ & - & $\operatorname{tr}$ & 6 & 12 & 11 & 2 & 4 & - & $\operatorname{tr}$ & $\operatorname{tr}$ & 45 & 10 & 1 & 2 & 3 & $\operatorname{tr}$ & - & 9 & - & 2 & & 3 & 32 & 4 & $\mathrm{ND}$ & $\operatorname{tr}$ \\
\hline 2-OH 14:0 & 10 & 14 & 14 & 13 & 10 & 13 & 10 & 10 & 16 & 18 & 17 & 11 & 7 & 2 & 9 & 8 & 17 & 16 & 28 & 18 & 20 & 13 & & & 14 & 3 & 18 & 23 & 10 \\
\hline 2-OH 15:0 & - & - & - & - & - & 2 & 4 & 3 & $\operatorname{tr}$ & 3 & - & - & - & 13 & - & - & $\operatorname{tr}$ & - & - & - & - & - & & & 2 & 15 & - & - & 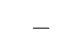 \\
\hline 2-OH 16:1 & - & - & $\operatorname{tr}$ & - & - & $\operatorname{tr}$ & - & - & - & - & - & - & - & - & $\operatorname{tr}$ & 6 & - & - & - & - & - & - & & & - & - & - & - & \\
\hline 2-OH 16:0 & 1 & 3 & 2 & - & - & $\operatorname{tr}$ & - & - & $\operatorname{tr}$ & - & 7 & - & 4 & $\operatorname{tr}$ & 2 & 4 & $\operatorname{tr}$ & - & - & - & - & - & & & 2 & - & - & - & \\
\hline \multicolumn{30}{|l|}{ Lipids: } \\
\hline SGL-1' & - & - & + & + & - & - & - & - & - & - & - & - & + & + & - & - & - & - & - & - & - & - & & & - & - & - & - & \\
\hline Other SGL(s)* & - & - & - & - & - & - & - & - & + & + & - & + & - & - & - & - & + & - & + & + & + & + & & & + & + & + & + & \\
\hline \multicolumn{29}{|l|}{ Major polyamines:† } & \\
\hline Spermidine & $+{ }^{a}$ & $+^{a}$ & $+{ }^{a}$ & & $+^{b}$ & $+{ }^{a}$ & $+{ }^{a}$ & $+^{a}$ & $+{ }^{a}$ & $+^{a}$ & $+{ }^{a}$ & $+{ }^{a}$ & $+{ }^{a}$ & $+^{a}$ & $+^{c, d}$ & $+{ }^{a}$ & & & & $+^{c}$ & & & & & & & & & \\
\hline Homospermidine & & & & & & & & & $+^{c}$ & & & & & $+{ }^{d}$ & & & $+{ }^{a}$ & & $+{ }^{a}$ & $+{ }^{a}$ & & & & & $+{ }^{a}$ & $+^{a}$ & $+{ }^{a}$ & $+^{a}$ & \\
\hline
\end{tabular}

* Other SGL(s) represents SGL-2, -3 or -4 .

$\dagger$ Data were taken from: a, Takeuchi et al. (2001); b. Stolz et al. (2000); c, Busse \& Auling (1988); d, Segers et al. (1994); e, Hamana \& Matsuzaki (1993). 
Table 6. Antimicrobial susceptibility testing of 28 type strains of Sphingomonas species determined by the Kirby-Bauer method

See Table 2 for identities of strains. Abbreviations: AMPC, amoxicillin; CVC, clavulanic acid; S, susceptible; IM, intermediate; R, resistant. Except for the combinations $\mathrm{AMPC} / \mathrm{CVC}$ and sulfamethoxazole/trimethoprim, discs contained a single agent.

\begin{tabular}{|c|c|c|c|c|c|c|c|c|c|c|c|c|c|c|c|c|c|c|c|c|c|c|c|c|c|c|c|c|}
\hline Antimicrobial agent (mg/disc) & 1 & 2 & 3 & 4 & 5 & 6 & 7 & 8 & 9 & 10 & 11 & 12 & 13 & 14 & 15 & 16 & 17 & 18 & 19 & 20 & 21 & 22 & 23 & 24 & 25 & 26 & 27 & 28 \\
\hline AMPC/CVC $(20 / 10)$ & $\mathrm{s}$ & $\mathrm{s}$ & $\mathrm{s}$ & IM & $\mathrm{s}$ & $\mathrm{s}$ & $\mathrm{s}$ & $\mathrm{s}$ & $\mathrm{s}$ & $\mathrm{s}$ & $\mathrm{s}$ & $\mathrm{s}$ & IM & $\mathrm{s}$ & $\mathrm{s}$ & $\mathrm{s}$ & $\mathrm{s}$ & $\mathrm{s}$ & $\mathrm{s}$ & $\mathrm{s}$ & $\mathrm{s}$ & $\mathrm{s}$ & $\mathrm{s}$ & $\mathrm{R}$ & $\mathrm{s}$ & $\mathrm{s}$ & $\mathrm{s}$ & $\mathrm{s}$ \\
\hline Amoxicillin (25) & $\mathrm{s}$ & $\mathrm{R}$ & $\mathrm{s}$ & $\mathrm{R}$ & $\mathrm{R}$ & $\mathrm{s}$ & $\mathrm{s}$ & $\mathrm{s}$ & $\mathrm{s}$ & $\mathrm{s}$ & $\mathrm{s}$ & $\mathrm{R}$ & $\mathrm{R}$ & $\mathrm{s}$ & $\mathrm{S}$ & $\mathrm{s}$ & $\mathrm{R}$ & $\mathrm{s}$ & $\mathrm{R}$ & $\mathrm{s}$ & $\mathrm{R}$ & $\mathrm{S}$ & $\mathrm{s}$ & $\mathrm{R}$ & $\mathrm{s}$ & $\mathrm{s}$ & $\mathrm{s}$ & $\mathrm{s}$ \\
\hline Penicillin G (10) & $\mathrm{s}$ & $\mathrm{R}$ & $\mathrm{s}$ & $\mathrm{R}$ & $\mathrm{R}$ & $\mathrm{s}$ & $\mathrm{s}$ & $\mathrm{R}$ & $\mathrm{s}$ & $\mathrm{s}$ & $\mathrm{s}$ & $\mathrm{R}$ & $\mathrm{R}$ & $\mathrm{s}$ & $\mathrm{s}$ & $\mathrm{s}$ & $\mathrm{R}$ & $\mathrm{R}$ & $\mathrm{R}$ & $\mathrm{s}$ & $\mathrm{s}$ & $\mathrm{R}$ & $\mathrm{R}$ & $\mathrm{R}$ & $\mathrm{s}$ & $\mathrm{R}$ & $\mathrm{s}$ & $\mathrm{s}$ \\
\hline Ampicillin (10) & $\mathrm{s}$ & $\mathrm{R}$ & $\mathrm{s}$ & $\mathrm{R}$ & $\mathrm{R}$ & $\mathrm{s}$ & $\mathrm{s}$ & $\mathrm{s}$ & $\mathrm{s}$ & $\mathrm{s}$ & $\mathrm{s}$ & $\mathrm{R}$ & $\mathrm{R}$ & $\mathrm{s}$ & $\mathrm{s}$ & $\mathrm{s}$ & $\mathrm{R}$ & $\mathrm{s}$ & $\mathrm{R}$ & $\mathrm{s}$ & $\mathrm{R}$ & $\mathrm{s}$ & $\mathrm{s}$ & $\mathrm{R}$ & $\mathrm{s}$ & $\mathrm{s}$ & $\mathrm{s}$ & $\mathrm{s}$ \\
\hline Piperacillin (100) & $\mathrm{s}$ & $\mathrm{R}$ & $\mathrm{R}$ & $\mathrm{R}$ & $\mathrm{R}$ & $\mathrm{s}$ & $\mathrm{s}$ & $\mathrm{s}$ & IM & $\mathrm{s}$ & $\mathrm{s}$ & $\mathrm{R}$ & $\mathrm{R}$ & $\mathrm{s}$ & $\mathrm{s}$ & $\mathrm{s}$ & $\mathrm{R}$ & $\mathrm{R}$ & $\mathrm{R}$ & $\mathrm{R}$ & $\mathrm{R}$ & $\mathrm{R}$ & $\mathrm{R}$ & $\mathrm{R}$ & $\mathrm{s}$ & IM & $\mathrm{s}$ & $\mathrm{s}$ \\
\hline Cefoperazon (75) & $\mathrm{s}$ & $\mathrm{R}$ & $\mathrm{s}$ & $\mathrm{R}$ & $\mathrm{R}$ & $\mathrm{S}$ & $\mathrm{s}$ & $\mathrm{s}$ & IM & $\mathrm{S}$ & $\mathrm{s}$ & $\mathrm{R}$ & $\mathrm{R}$ & $\mathrm{S}$ & $\mathrm{s}$ & $\mathrm{S}$ & $\mathrm{R}$ & $\mathrm{R}$ & $\mathrm{R}$ & $\mathrm{s}$ & $\mathrm{R}$ & $\mathrm{R}$ & $\mathrm{R}$ & $\mathrm{R}$ & $\mathrm{R}$ & $\mathrm{S}$ & $\mathrm{s}$ & IM \\
\hline Moxalactam (30) & $\mathrm{R}$ & $\mathrm{R}$ & $\mathrm{R}$ & $\mathrm{s}$ & $\mathrm{R}$ & $\mathrm{s}$ & $\mathrm{R}$ & $\mathrm{R}$ & IM & $\mathrm{s}$ & $\mathrm{R}$ & $\mathrm{R}$ & $\mathrm{R}$ & $\mathrm{R}$ & $\mathrm{R}$ & $\mathrm{R}$ & $\mathrm{R}$ & $\mathrm{R}$ & $\mathrm{R}$ & $\mathrm{R}$ & $\mathrm{s}$ & $\mathrm{R}$ & $\mathrm{R}$ & IM & IM & $\mathrm{R}$ & $\mathrm{s}$ & $\mathrm{R}$ \\
\hline Cefaclor (30) & $\mathrm{s}$ & $\mathrm{R}$ & $\mathrm{s}$ & $\mathrm{s}$ & $\mathrm{S}$ & $\mathrm{s}$ & IM & IM & $\mathrm{s}$ & $\mathrm{s}$ & $\mathrm{R}$ & $\mathrm{s}$ & $\mathrm{R}$ & IM & $\mathrm{s}$ & $\mathrm{s}$ & $\mathrm{R}$ & $\mathrm{s}$ & $\mathrm{R}$ & $\mathrm{s}$ & IM & $\mathrm{s}$ & $\mathrm{s}$ & $\mathrm{R}$ & $\mathrm{S}$ & $\mathrm{s}$ & $\mathrm{s}$ & $\mathrm{s}$ \\
\hline Ceftazidime (30) & $\mathrm{s}$ & $\mathrm{s}$ & $\mathrm{s}$ & $\mathrm{R}$ & $\mathrm{s}$ & $\mathrm{s}$ & $\mathrm{s}$ & $\mathrm{s}$ & $\mathrm{s}$ & $\mathrm{s}$ & $\mathrm{IM}$ & $\mathrm{R}$ & $\mathrm{R}$ & $\mathrm{s}$ & $\mathrm{s}$ & $\mathrm{s}$ & $\mathrm{R}$ & $\mathrm{s}$ & $\mathrm{R}$ & $\mathrm{s}$ & $\mathrm{R}$ & IM & $\mathrm{R}$ & $\mathrm{s}$ & $\mathrm{s}$ & $\mathrm{s}$ & $\mathrm{s}$ & $\mathrm{s}$ \\
\hline Flomoxef (30) & $\mathrm{s}$ & $\mathrm{s}$ & $\mathrm{R}$ & $\mathrm{R}$ & s & $\mathrm{s}$ & s & $\mathrm{s}$ & $\mathrm{s}$ & $\mathrm{s}$ & $\mathrm{IM}$ & $\mathrm{R}$ & $\mathrm{R}$ & $\mathrm{R}$ & IM & IM & $\mathrm{R}$ & $\mathrm{s}$ & $\mathrm{R}$ & $\mathrm{s}$ & $\mathrm{R}$ & $\mathrm{R}$ & $\mathrm{R}$ & IM & $\mathrm{R}$ & $\mathrm{R}$ & $\mathrm{s}$ & $\mathrm{s}$ \\
\hline Cefazolin (30) & $\mathrm{R}$ & $\mathrm{R}$ & $\mathrm{R}$ & $\mathrm{R}$ & $\mathrm{R}$ & $\mathrm{s}$ & $\mathrm{R}$ & $\mathrm{R}$ & $\mathrm{s}$ & $\mathrm{s}$ & $\mathrm{R}$ & $\mathrm{R}$ & $\mathrm{R}$ & $\mathrm{s}$ & $\mathrm{R}$ & $\mathrm{s}$ & $\mathrm{R}$ & $\mathrm{s}$ & $\mathrm{R}$ & $\mathrm{R}$ & $\mathrm{R}$ & $\mathrm{R}$ & $\mathrm{R}$ & $\mathrm{R}$ & $\mathrm{R}$ & $\mathrm{R}$ & $\mathrm{s}$ & $\mathrm{s}$ \\
\hline Cefmetazole (30) & $\mathrm{s}$ & $\mathrm{s}$ & $\mathrm{R}$ & $\mathrm{s}$ & $\mathrm{S}$ & $\mathrm{s}$ & $\mathrm{R}$ & $\mathrm{R}$ & $\mathrm{s}$ & $\mathrm{s}$ & $\mathrm{R}$ & $\mathrm{R}$ & $\mathrm{R}$ & $\mathrm{R}$ & IM & IM & $\mathrm{R}$ & $\mathrm{s}$ & $\mathrm{R}$ & $\mathrm{R}$ & $\mathrm{s}$ & $\mathrm{R}$ & $\mathrm{R}$ & $\mathrm{R}$ & $\mathrm{s}$ & IM & $\mathrm{s}$ & $\mathrm{s}$ \\
\hline Cefotaxime (30) & $\mathrm{s}$ & $\mathrm{s}$ & $\mathrm{s}$ & $\mathrm{s}$ & $\mathrm{s}$ & $\mathrm{s}$ & $\mathrm{s}$ & $\mathrm{s}$ & $\mathrm{s}$ & $\mathrm{s}$ & $\mathrm{s}$ & $\mathrm{s}$ & $\mathrm{R}$ & $\mathrm{s}$ & $\mathrm{s}$ & $\mathrm{s}$ & $\mathrm{R}$ & $\mathrm{s}$ & $\mathrm{R}$ & $\mathrm{s}$ & $\mathrm{s}$ & IM & $\mathrm{s}$ & IM & $\mathrm{s}$ & $\mathrm{s}$ & $\mathrm{s}$ & $\mathrm{s}$ \\
\hline Meropenem (10) & $\mathrm{s}$ & $\mathrm{s}$ & $\mathrm{s}$ & $\mathrm{R}$ & $\mathrm{s}$ & $\mathrm{s}$ & $\mathrm{s}$ & $\mathrm{s}$ & $\mathrm{s}$ & $\mathrm{s}$ & IM & $\mathrm{R}$ & $\mathrm{R}$ & IM & $\mathrm{s}$ & $\mathrm{s}$ & $\mathrm{s}$ & $\mathrm{s}$ & $\mathrm{s}$ & $\mathrm{s}$ & $\mathrm{R}$ & $\mathrm{s}$ & $\mathrm{s}$ & $\mathrm{s}$ & IM & $\mathrm{s}$ & $\mathrm{s}$ & $\mathrm{s}$ \\
\hline Aztreonam (30) & $\mathrm{R}$ & IM & $\mathrm{R}$ & $\mathrm{s}$ & IM & $\mathrm{R}$ & IM & $\mathrm{s}$ & $\mathrm{R}$ & $\mathrm{s}$ & $\mathrm{S}$ & $\mathrm{s}$ & $\mathrm{R}$ & $\mathrm{R}$ & $\mathrm{R}$ & IM & $\mathrm{R}$ & $\mathrm{R}$ & $\mathrm{R}$ & IM & $\mathrm{s}$ & $\mathrm{R}$ & $\mathrm{R}$ & $\mathrm{R}$ & $\mathrm{S}$ & $\mathrm{R}$ & $\mathrm{R}$ & $\mathrm{R}$ \\
\hline Imipenem (10) & $\mathrm{s}$ & $\mathrm{s}$ & $\mathrm{s}$ & $\mathrm{R}$ & $\mathrm{s}$ & $\mathrm{s}$ & $\mathrm{s}$ & $\mathrm{s}$ & $\mathrm{s}$ & $\mathrm{s}$ & $\mathrm{s}$ & $\mathrm{s}$ & $\mathrm{s}$ & $\mathrm{S}$ & $\mathrm{S}$ & $\mathrm{S}$ & $\mathrm{s}$ & $\mathrm{s}$ & IM & $\mathrm{s}$ & $\mathrm{R}$ & $\mathrm{s}$ & $\mathrm{s}$ & $\mathrm{s}$ & $\mathrm{S}$ & $\mathrm{s}$ & $\mathrm{s}$ & $\mathrm{s}$ \\
\hline Carumonam (30) & $\mathrm{R}$ & $\mathrm{R}$ & $\mathrm{R}$ & $\mathrm{s}$ & IM & $\mathrm{s}$ & $\mathrm{s}$ & $\mathrm{s}$ & $\mathrm{R}$ & $\mathrm{s}$ & $\mathrm{IM}$ & $\mathrm{s}$ & $\mathrm{R}$ & $\mathrm{R}$ & $\mathrm{R}$ & $\mathrm{R}$ & $\mathrm{R}$ & IM & $\mathrm{R}$ & $\mathrm{s}$ & $\mathrm{s}$ & $\mathrm{R}$ & $\mathrm{R}$ & $\mathrm{s}$ & $\mathrm{s}$ & $\mathrm{R}$ & $\mathrm{R}$ & $\mathrm{s}$ \\
\hline Panipenem (10) & $\mathrm{s}$ & $\mathrm{s}$ & $\mathrm{s}$ & $\mathrm{s}$ & $\mathrm{s}$ & $\mathrm{s}$ & $\mathrm{s}$ & $\mathrm{s}$ & $\mathrm{s}$ & $\mathrm{s}$ & $\mathrm{IM}$ & $\mathrm{R}$ & $\mathrm{s}$ & $\mathrm{s}$ & $\mathrm{s}$ & $\mathrm{s}$ & $\mathrm{s}$ & $\mathrm{s}$ & $\mathrm{s}$ & $\mathrm{s}$ & $\mathrm{s}$ & IM & $\mathrm{s}$ & $\mathrm{s}$ & $\mathrm{s}$ & $\mathrm{s}$ & $\mathrm{s}$ & $\mathrm{s}$ \\
\hline Gentamicin (10) & $\mathrm{s}$ & $\mathrm{s}$ & $\mathrm{s}$ & $\mathrm{s}$ & $\mathrm{s}$ & $\mathrm{s}$ & $\mathrm{s}$ & $\mathrm{s}$ & $\mathrm{s}$ & $\mathrm{s}$ & $\mathrm{s}$ & $\mathrm{S}$ & $\mathrm{s}$ & $\mathrm{s}$ & $\mathrm{R}$ & $\mathrm{s}$ & $\mathrm{s}$ & $\mathrm{s}$ & $\mathrm{s}$ & $\mathrm{s}$ & $\mathrm{s}$ & $\mathrm{s}$ & $\mathrm{s}$ & $\mathrm{s}$ & $\mathrm{s}$ & $\mathrm{s}$ & $\mathrm{s}$ & $\mathrm{s}$ \\
\hline Amikacin (30) & $\mathrm{s}$ & $\mathrm{s}$ & $\mathrm{s}$ & $\mathrm{s}$ & s & $\mathrm{s}$ & $\mathrm{s}$ & $\mathrm{s}$ & $\mathrm{s}$ & $\mathrm{s}$ & $\mathrm{s}$ & $\mathrm{s}$ & $\mathrm{s}$ & $\mathrm{s}$ & $\mathrm{s}$ & $\mathrm{s}$ & $\mathrm{s}$ & $\mathrm{s}$ & $\mathrm{s}$ & $\mathrm{s}$ & $\mathrm{s}$ & $\mathrm{s}$ & $\mathrm{s}$ & $\mathrm{s}$ & $\mathrm{s}$ & $\mathrm{s}$ & $\mathrm{s}$ & $\mathrm{s}$ \\
\hline Dibekacin (30) & $\mathrm{s}$ & $\mathrm{s}$ & $\mathrm{s}$ & $\mathrm{R}$ & $\mathrm{s}$ & $\mathrm{s}$ & $\mathrm{s}$ & $\mathrm{s}$ & $\mathrm{s}$ & $\mathrm{s}$ & $\mathrm{s}$ & $\mathrm{s}$ & $\mathrm{s}$ & $\mathrm{s}$ & $\mathrm{R}$ & $\mathrm{s}$ & $\mathrm{s}$ & $\mathrm{s}$ & $\mathrm{s}$ & $\mathrm{s}$ & $\mathrm{s}$ & $\mathrm{s}$ & $\mathrm{s}$ & $\mathrm{s}$ & $\mathrm{s}$ & $\mathrm{s}$ & $\mathrm{s}$ & $\mathrm{s}$ \\
\hline Clarithromycin (15) & $\mathrm{R}$ & $\mathrm{s}$ & $\mathrm{s}$ & $\mathrm{R}$ & $\mathrm{s}$ & $\mathrm{s}$ & $\mathrm{s}$ & $\mathrm{s}$ & $\mathrm{s}$ & $\mathrm{s}$ & $\mathrm{s}$ & $\mathrm{s}$ & $\mathrm{s}$ & $\mathrm{R}$ & $\mathrm{s}$ & $\mathrm{S}$ & $\mathrm{s}$ & $\mathrm{s}$ & $\mathrm{s}$ & $\mathrm{s}$ & IM & $\mathrm{s}$ & $\mathrm{s}$ & $\mathrm{R}$ & IM & $\mathrm{s}$ & $\mathrm{s}$ & $\mathrm{R}$ \\
\hline Roxithromycin (15) & $\mathrm{R}$ & $\mathrm{s}$ & $\mathrm{R}$ & $\mathrm{R}$ & IM & IM & IM & $\mathrm{s}$ & $\mathrm{R}$ & $\mathrm{S}$ & $\mathrm{R}$ & IM & IM & $\mathrm{R}$ & $\mathrm{R}$ & $\mathrm{S}$ & $\mathrm{s}$ & $\mathrm{S}$ & $\mathrm{s}$ & $\mathrm{s}$ & IM & $\mathrm{R}$ & $\mathrm{R}$ & $\mathrm{R}$ & IM & $\mathrm{R}$ & $\mathrm{R}$ & $\mathrm{R}$ \\
\hline Erythromycin (15) & $\mathrm{R}$ & $\mathrm{s}$ & IM & $\mathrm{S}$ & IM & $\mathrm{s}$ & IM & IM & IM & $\mathrm{s}$ & $\mathrm{IM}$ & IM & $\mathrm{s}$ & IM & IM & $\mathrm{S}$ & $\mathrm{s}$ & $\mathrm{s}$ & $\mathrm{s}$ & $\mathrm{s}$ & $\mathrm{s}$ & IM & $\mathrm{R}$ & $\mathrm{R}$ & $\mathrm{S}$ & $\mathrm{R}$ & $\mathrm{R}$ & $\mathrm{R}$ \\
\hline Tetracycline (30) & $\mathrm{s}$ & $\mathrm{s}$ & $\mathrm{s}$ & $\mathrm{s}$ & IM & $\mathrm{s}$ & $\mathrm{s}$ & $\mathrm{s}$ & $\mathrm{s}$ & $\mathrm{s}$ & $\mathrm{s}$ & $\mathrm{s}$ & $\mathrm{s}$ & $\mathrm{s}$ & $\mathrm{s}$ & $\mathrm{s}$ & $\mathrm{s}$ & $\mathrm{s}$ & $\mathrm{s}$ & $\mathrm{s}$ & $\mathrm{s}$ & $\mathrm{s}$ & $\mathrm{s}$ & $\mathrm{s}$ & $\mathrm{s}$ & $\mathrm{s}$ & $\mathrm{s}$ & IM \\
\hline Doxycycline (10) & $\mathrm{s}$ & $\mathrm{s}$ & $\mathrm{s}$ & $\mathrm{s}$ & $\mathrm{s}$ & $\mathrm{s}$ & $\mathrm{s}$ & $\mathrm{s}$ & $\mathrm{s}$ & $\mathrm{s}$ & $\mathrm{s}$ & $\mathrm{s}$ & $\mathrm{s}$ & $\mathrm{s}$ & $\mathrm{s}$ & $\mathrm{s}$ & $\mathrm{s}$ & $\mathrm{s}$ & $\mathrm{s}$ & $\mathrm{s}$ & $\mathrm{s}$ & $\mathrm{s}$ & $\mathrm{s}$ & $\mathrm{s}$ & $\mathrm{s}$ & $\mathrm{s}$ & $\mathrm{s}$ & $\mathrm{s}$ \\
\hline Minocycline (30) & $\mathrm{s}$ & $\mathrm{s}$ & $\mathrm{s}$ & $\mathrm{s}$ & $\mathrm{s}$ & $\mathrm{s}$ & $\mathrm{s}$ & $\mathrm{s}$ & $\mathrm{s}$ & $\mathrm{s}$ & $\mathrm{s}$ & $\mathrm{s}$ & $\mathrm{S}$ & $\mathrm{S}$ & $\mathrm{S}$ & $\mathrm{S}$ & $\mathrm{s}$ & $\mathrm{s}$ & $\mathrm{s}$ & $\mathrm{s}$ & $\mathrm{R}$ & $\mathrm{s}$ & $\mathrm{s}$ & $\mathrm{R}$ & $\mathrm{s}$ & $\mathrm{s}$ & $\mathrm{s}$ & $\mathrm{s}$ \\
\hline Polymyxin B (300) & $\mathrm{s}$ & $\mathrm{s}$ & $\mathrm{s}$ & $\mathrm{s}$ & s & $\mathrm{s}$ & $\mathrm{s}$ & $\mathrm{R}$ & IM & $\mathrm{R}$ & $\mathrm{s}$ & $\mathrm{R}$ & $\mathrm{s}$ & $\mathrm{s}$ & IM & IM & IM & $\mathrm{s}$ & $\mathrm{R}$ & IM & $\mathrm{R}$ & $\mathrm{R}$ & $\mathrm{R}$ & $\mathrm{R}$ & $\mathrm{R}$ & $\mathrm{R}$ & $\mathrm{R}$ & IM \\
\hline Norfloxacin (10) & $\mathrm{s}$ & $\mathrm{s}$ & IM & $\mathrm{R}$ & $\mathrm{s}$ & $\mathrm{R}$ & $\mathrm{R}$ & $\mathrm{R}$ & $\mathrm{R}$ & $\mathrm{R}$ & $\mathrm{R}$ & $\mathrm{R}$ & $\mathrm{s}$ & IM & IM & $\mathrm{s}$ & $\mathrm{R}$ & $\mathrm{s}$ & $\mathrm{s}$ & IM & $\mathrm{R}$ & $\mathrm{R}$ & $\mathrm{R}$ & $\mathrm{R}$ & $\mathrm{R}$ & $\mathrm{R}$ & $\mathrm{R}$ & $\mathrm{R}$ \\
\hline Trimethoprim (5) & $\mathrm{R}$ & $\mathrm{R}$ & $\mathrm{R}$ & $\mathrm{S}$ & $\mathrm{R}$ & $\mathrm{R}$ & $\mathrm{R}$ & $\mathrm{R}$ & $\mathrm{R}$ & $\mathrm{R}$ & $\mathrm{R}$ & $\mathrm{R}$ & $\mathrm{s}$ & $\mathrm{R}$ & $\mathrm{R}$ & $\mathrm{R}$ & $\mathrm{R}$ & $\mathrm{s}$ & $\mathrm{R}$ & $\mathrm{R}$ & $\mathrm{S}$ & $\mathrm{R}$ & $\mathrm{R}$ & $\mathrm{R}$ & $\mathrm{R}$ & $\mathrm{R}$ & $\mathrm{R}$ & $\mathrm{R}$ \\
\hline Ciprofloxacin (5) & $\mathrm{s}$ & $\mathrm{s}$ & $\mathrm{s}$ & $\mathrm{s}$ & $\mathrm{s}$ & $\mathrm{s}$ & $\mathrm{R}$ & $\mathrm{R}$ & $\mathrm{R}$ & $\mathrm{R}$ & $\mathrm{R}$ & $\mathrm{R}$ & $\mathrm{R}$ & $\mathrm{S}$ & $\mathrm{S}$ & $\mathrm{S}$ & $\mathrm{s}$ & $\mathrm{s}$ & $\mathrm{s}$ & $\mathrm{s}$ & IM & IM & $\mathrm{R}$ & IM & $\mathrm{R}$ & IM & $\mathrm{s}$ & $\mathrm{R}$ \\
\hline Ofloxacin (5) & $\mathrm{s}$ & $\mathrm{s}$ & $\mathrm{s}$ & $\mathrm{s}$ & $\mathrm{S}$ & $\mathrm{s}$ & $\mathrm{R}$ & $\mathrm{R}$ & $\mathrm{R}$ & $\mathrm{R}$ & $\mathrm{R}$ & $\mathrm{R}$ & $\mathrm{s}$ & $\mathrm{s}$ & IM & $\mathrm{s}$ & $\mathrm{s}$ & $\mathrm{s}$ & $\mathrm{s}$ & $\mathrm{s}$ & $\mathrm{s}$ & IM & $\mathrm{s}$ & $\mathrm{R}$ & $\mathrm{R}$ & IM & $\mathrm{s}$ & $\mathrm{R}$ \\
\hline Tosufloxacin (5) & $\mathrm{s}$ & $\mathrm{s}$ & IM & $\mathrm{s}$ & IM & $\mathrm{s}$ & $\mathrm{R}$ & $\mathrm{R}$ & $\mathrm{R}$ & $\mathrm{R}$ & $\mathrm{R}$ & $\mathrm{R}$ & $\mathrm{s}$ & IM & IM & $\mathrm{R}$ & $\mathrm{s}$ & $\mathrm{s}$ & $\mathrm{s}$ & $\mathrm{s}$ & $\mathrm{s}$ & $\mathrm{s}$ & IM & $\mathrm{s}$ & IM & IM & $\mathrm{R}$ & $\mathrm{R}$ \\
\hline Levofloxacin (5) & $\mathrm{s}$ & $\mathrm{s}$ & $\mathrm{s}$ & $\mathrm{s}$ & $\mathrm{s}$ & $\mathrm{s}$ & IM & $\mathrm{R}$ & $\mathrm{s}$ & $\mathrm{R}$ & $\mathrm{R}$ & $\mathrm{R}$ & $\mathrm{s}$ & $\mathrm{s}$ & IM & $\mathrm{s}$ & $\mathrm{s}$ & $\mathrm{s}$ & $\mathrm{s}$ & $\mathrm{s}$ & $\mathrm{s}$ & $\mathrm{s}$ & $\mathrm{s}$ & $\mathrm{s}$ & $\mathrm{R}$ & $\mathrm{s}$ & $\mathrm{s}$ & $\mathrm{R}$ \\
\hline Sparfloxacin (5) & $\mathrm{s}$ & $\mathrm{s}$ & $\mathrm{s}$ & $\mathrm{s}$ & s & $\mathrm{s}$ & $\mathrm{R}$ & IM & IM & $\mathrm{s}$ & $\mathrm{R}$ & $\mathrm{R}$ & $\mathrm{s}$ & $\mathrm{s}$ & IM & $\mathrm{s}$ & $\mathrm{s}$ & $\mathrm{s}$ & $\mathrm{s}$ & $\mathrm{s}$ & $\mathrm{s}$ & $\mathrm{s}$ & $\mathrm{s}$ & $\mathrm{s}$ & IM & $\mathrm{s}$ & $\mathrm{R}$ & $\mathrm{R}$ \\
\hline $\begin{array}{l}\text { Sulfamethoxazole/trimethoprim } \\
(23 \cdot 8 / 1 \cdot 25)\end{array}$ & $\mathrm{s}$ & $\mathrm{R}$ & $\mathrm{s}$ & $\mathrm{s}$ & $\mathrm{R}$ & $\mathrm{s}$ & IM & $\mathrm{R}$ & $\mathrm{s}$ & $\mathrm{R}$ & $\mathrm{R}$ & $\mathrm{R}$ & $\mathrm{s}$ & $\mathrm{R}$ & $\mathrm{R}$ & $\mathrm{s}$ & $\mathrm{s}$ & $\mathrm{s}$ & $\mathrm{s}$ & $\mathrm{s}$ & IM & $\mathrm{R}$ & $\mathrm{s}$ & $\mathrm{R}$ & $\mathrm{s}$ & $\mathrm{R}$ & IM & $\mathrm{R}$ \\
\hline Summary: & & & & & & & & & & & & & & & & & & & & & & & & & & & & \\
\hline Number of agents resisted & 8 & 11 & 9 & 14 & 9 & 3 & 9 & 12 & 8 & 8 & 13 & 21 & 16 & 9 & 9 & 4 & 16 & 5 & 16 & 5 & 12 & 14 & 16 & 19 & 9 & 12 & 9 & 13 \\
\hline Percentage resisted & 22 & 31 & 25 & 39 & 25 & 8 & 25 & 33 & 22 & 22 & 36 & 58 & 44 & 25 & 25 & 11 & 44 & 14 & 44 & 14 & 33 & 39 & 44 & 53 & 25 & 33 & 25 & 36 \\
\hline Number scored as $\mathrm{S}+\mathrm{IM}$ & 28 & 25 & 27 & 22 & 16 & 33 & 27 & 24 & 28 & 28 & 23 & 15 & 20 & 27 & 27 & 32 & 20 & 31 & 20 & 31 & 24 & 22 & 20 & 17 & 27 & 24 & 27 & 23 \\
\hline
\end{tabular}


Sphingomonas ursincola, Sphingomonas natatoria, Sphingomonas roseiflava, Sphingomonas pruni and Sphingomonas asaccharolytica are non-motile, since they do not show spreading growth on $0.3 \%$ semi-solid agar plates. Strictly aerobic. Metabolism is respiratory, but not fermentative. Cells of Sphingomonas ursincola and Sphingomonas natatoria contain bacteriochlorophyll $a$ and these species are facultative photoorganotrophs. Colony colour varies from species to species, for instance, deep-yellow, orange, lemonyellow or non-pigmented. The deep-yellow pigment of Sphingomonas paucimobilis is reported to be a carotenoid, nostoxanthin, rather than a xanthomonadin. This pigment was also found in Sphingomonas [Pseudomonas] echinoides (Denner et al., 1999) and Sphingomonas sanguinis [Pseudomonas paucimobilis] (Jenkins \& Starr, 1985; Starr et al., 1977). In certain cases, pigmentation differs depending on the medium used. Colonies of some non-pigmented strains such as Sphingomonas yanoikuyae become lemon-yellow after more than 3 days incubation at room temperature. The type strain of Sphingomonas herbicidovorans produces alcapton via accumulation of homogentisic acid, as observed in pyomelanogenic strains of Pseudomonas aeruginosa (Gessard, 1891; Mann, 1979; Yabuuchi \& Ohyama, 1972).

Catalase is produced. Oxidase-positive with a few exceptions. The type strain of Sphingomonas herbicidovorans produces alcapton from phenylalanine (Zipper et al., 1996) and a water-soluble melanin-like brown pigment is produced in peptone media. Respiratory quinone is ubiquinone 10. SGL-1 and 2-hydroxymyristic acid, but not 3-hydroxy fatty acids, are found in cellular lipids of all 28 species examined. Major long-chain bases of SGL-1 are C-18:0, C-20:1 and C-21 cyclopropane. SGL-1' exists in cellular lipids of Sphingomonas yanoikuyae, Sphingomonas macrogoltabidus, Sphingomonas terrae, Sphingomonas wittichii and Sphingomonas cloacae. The chemical structure, function and distribution of SGLs of Sphingomonas species have been reported from other members of the Proteobacteria (Kawahara et al., 1999). The type strain of Sphingomonas herbicidovorans represents the second bacterial species in which homogentisic acid accumulates through metabolic defects in phenylalanine and tyrosine metabolism.

At the time of writing, the 33 species listed in Table 1 are assigned to the genus Sphingomonas. The 'Bacterial Nomenclature Up to Date' web site at the DSMZ (http://www.dsmz.de) should be referred to for further information.

Isolated from a wide variety of natural and artificial environments. Some are pathogenic for humans or lettuce. The plant-pathogenic species Sphingomonas suberifaciens is susceptible to 33 of 36 Sensi-discs; clinical and hospital-environment isolates are susceptible to fluoroquinolones, whereas species from other sources, especially from soil, are generally resistant to various kinds of antimicrobial agents. Strains of species with optimal growth temperatures of 26 or $28^{\circ} \mathrm{C}$ are unlikely to be human pathogens, although organisms in the natural environment that are resistant to various kinds of antimicrobials are potential human pathogens. Strains of certain species metabolize dioxins (Wittich et al., 1992; Nohynek et al., 1995, 1996) and other chlorophenol derivatives (Wittich et al., 1992). The $\mathrm{G}+\mathrm{C}$ content of DNA ranges from 59 to $67 \mathrm{~mol} \%$ (HPLC). The type species is Sphingomonas paucimobilis (Holmes et al. 1977) Yabuuchi et al. 1990.

\section{ACKNOWLEDGEMENTS}

This work was supported by grants from Ministry of Health, Labour and Welfare (Research on Environmental Health, H11-Seikatsu-015), Research on Emerging and Re-emerging Infectious Diseases (Health Sciences Research Grants), Ministry of the Environment (Global Environment Research Fund), Ministry of Education, Culture, Sports, Science and Technology and The United States-Japan Cooperative Medical Science Program against Tuberculosis and Leprosy.

\section{REFERENCES}

Balkwill, D. L., Drake, G. R., Reeves, R. H. \& 7 other authors (1997). Taxonomic study of aromatic-degrading bacteria from deep-terrestrialsubsurface sediments and description of Sphingomonas aromaticivorans sp. nov., Sphingomonas subterranea sp. nov., and Sphingomonas stygia sp. nov. Int J Syst Bacteriol 47, 191-201.

Bory, C., Boulieu, R., Chantin, C. \& Mathieu, M. (1990). Diagnosis of alcaptonuria: rapid analysis of homogentisic acid by HPLC. Clin Chim Acta 189, 7-11.

Busse, H. J. \& Auling, G. (1988). Polyamine pattern as a chemotaxonomic marker within the Proteobacteria. Syst Appl Microbiol 11, $1-8$.

Denner, E. B. M., Kämpfer, P., Busse, H.-J. \& Moore, E. R. B. (1999). Reclassification of Pseudomonas echinoides Heumann 1962, 343 ${ }^{\mathrm{AL}}$, in the genus Sphingomonas as Sphingomonas echinoides comb. nov. Int $J$ Syst Bacteriol 49, 1103-1109.

Gessard, C. (1891). Variété mélanogène du bacillus pyocyanique. Ann Inst Pasteur 15, 817-831.

Hamana, K. \& Matsuzaki, S. (1993). Polyamine distribution patterns serve as a phenotypic marker in the chemotaxonomy of the Proteobacteria. Can J Microbiol 39, 304-310.

Hanada, S., Kawase, Y., Hiraishi, A., Takaichi, S., Matsuura, K., Shimada, K. \& Nagashima, K. V. P. (1997). Porphyrobacter tepidarius sp. nov., a moderately thermophilic aerobic photosynthetic bacterium isolated from a hot spring. Int J Syst Bacteriol 47, 408-413.

Hiraishi, A., Kuraishi, H. \& Kawahara, K. (2000). Emendation of the description of Blastomonas natatoria (Sly 1985) Sly and Cahill 1997 as an aerobic photosynthetic bacterium and reclassification of Erythromonas ursincola Yurkov et al. 1997 as Blastomonas ursincola comb. nov. Int J Syst Evol Microbiol 50, 1113-1118.

Jenkins, C. L. \& Starr, M. P. (1985). Formation of halogenated arylpolyene (xanthomonadin) pigments by the type and other yellowpigmented strains of Xanthomonas maltophilia. Ann Inst Pasteur Microbiol 136B, 257-264.

Jenkins, C. L., Andrewes, A. G., McQuade, T. J. \& Starr, M. P. (1979). The pigment of Pseudomonas paucimobilis is a carotenoid (nostoxanthin), rather than a brominated aryl-polyene (xanthomonadin). Curr Microbiol 48, 1-4.

Kämpfer, P., Denner, E. B. M., Meyer, S., Moore, E. R. B. \& Busse, H.-J. (1997). Classification of "Pseudomonas azotocolligans" Anderson 1955, 132, in the genus Sphingomonas as Sphingomonas trueperi sp. nov. Int J Syst Bacteriol 47, 577-583. 
Kawahara, K., Kuraishi, H. \& Zähringer, U. (1999). Chemical structure and function of glycosphingolipids of Sphingomonas spp. and their distribution among members of the alpha-4 subclass of Proteobacteria. J Ind Microbiol Biotechnol 23, 408-413.

Kimura, M. (1980). A simple method for estimating evolutionary rates of base substitutions through comparative studies of nucleotide sequences. $J$ Mol Evol 16, 111-120.

Kondratieva, E. N., Pfenning, N. \& Trüper, H. G. (1992). The phototrophic prokaryotes. In The Prokaryotes: A Handbook on the Biology of Bacteria. Ecophysiology, Isolation, Identification, Applications, 2nd edn, pp. 312-330. Edited by A. Balows, H. G. Trüper, M. Dworkin, W. Harder \& K. H. Schleifer. New York: Springer.

Kosako, Y., Yabuuchi, E., Naka, T., Fujiwara, N. \& Kobayashi, K. (2000). Proposal of Sphingomonadaceae fam. nov. consisted of Sphingomonas Yabuuchi et al. 1990, Erythrobacter Shiba and Shimidu 1982, Erythromicrobium Yurkov et al. 1994, Porphyrobacter Fuerst et al. 1993, Zymomonas Kluyver and van Niel 1936, and Sandaracinobacter Yurkov et al. 1997, with the type genus Sphingomonas Yabuuchi et al. 1990. Microbiol Immunol 44, 563-575.

Mann, S. (1979). Über melaninbildende Stämme von Pseudomonas aeruginosa. Arch Mikrobiol 65, 359-379.

Mathieu, P., Prevel, A., David, L. \& 8 other authors (1997). Screening infants for neuroblastoma: discovery of alkaptonuria in one case. Clin Chim Acta 264, 255-259.

Naka, T., Fujiwara, N., Yabuuchi, E., Doe, M., Kobayashi, K., Kato, Y. \& Yano, I. (2000). A novel sphingoglycolipid containing galacturonic acid and 2-hydroxy fatty acid in cellular lipids of Sphingomonas yanoikuyae. J Bacteriol 182, 2660-2663.

Nohynek, L. J., Suhonen, E. L., Nurmiaho-Lassila, E.-L., Hantula, J. \& Salkinoja-Salonen, M. (1995). Description of four pentachlorophenol-degrading bacterial strains as Sphingomonas chlorophenolica sp. nov. Syst Appl Microbiol 18, 527-538.

Nohynek, L. J., Nurmiaho-Lassila, E.-L., Suhonen, E. L., Busse, H.-J., Mohammadi, M., Hantula, J., Rainey, F. \& Salkinoja-Salonen, M. S. (1996). Description of chlorophenol-degrading Pseudomonas sp. strains $\mathrm{KF}^{\mathrm{T}}, \mathrm{KF} 3$, and NKF1 as a new species of the genus Sphingomonas, Sphingomonas subarctica sp. nov. Int J Syst Bacteriol 46, 1042-1055.

Saitou, N. \& Nei, M. (1987). The neighbor-joining method: a new method for reconstructing phylogenetic trees. Mol Biol Evol 4, 406-425.

Segers, R., Vancanneyt, M., Pot, B., Torck, U., Hoste, B., Dewettinck, D., Falsen, E., Kersters, K. \& De Vos, P. (1994). Classification of Pseudomonas diminuta Leifson and Hugh 1954 and Pseudomonas vesicularis Büsing, Döll, and Freytag 1953 in Brevundimonas gen. nov. as Brevundimonas diminuta comb. nov. and Brevundimonas vesicularis comb. nov., respectively. Int J Syst Bacteriol 44, 499-510.

Sneath, P. H. A. \& Sokal, R. R. (1973). Numerical Taxonomy: the Principles and Practice of Numerical Classification. San Francisco: W. H. Freeman.

Starr, M. P., Jenkins, C. L., Bussey, L. B. \& Andrewes, A. G. (1977). Chemotaxonomic significance of the xanthomonadins, novel brominated aryl-polyene pigments produced by bacteria of the genus Xanthomonas. Arch Microbiol 113, 1-9.

Stolz, A., Schmidt-Maag, C., Denner, E. B. M., Busse, H.-J., Egli, T. \& Kämpfer, P. (2000). Description of Sphingomonas xenophaga sp. nov. for strains $\mathrm{BN}^{\mathrm{T}}$ and $\mathrm{N}, \mathrm{N}$ which degrade xenobiotic aromatic compounds. Int J Syst Evol Microbiol 50, 35-41.
Takeuchi, M., Sawada, H., Oyaizu, H. \& Yokota, A. (1994). Phylogenetic evidence for Sphingomonas and Rhizomonas as nonphotosynthetic members of the alpha-4 subclass of the Proteobacteria. Int J Syst Bacteriol 44, 308-314.

Takeuchi, M., Sakane, T., Yanagi, M., Yamasato, K., Hamana, K. \& Yokota, A. (1995). Taxonomic study of bacteria isolated from plants: proposal of Sphingomonas rosa sp. nov., Sphingomonas pruni sp. nov., Sphingomonas asaccharolytica sp. nov., and Sphingomonas mali sp. nov. Int J Syst Bacteriol 45, 334-341.

Takeuchi, M., Hamana, K. \& Hiraishi, A. (2001). Proposal of the genus Sphingomonas sensu stricto and three new genera, Sphingobium, Novosphingobium and Sphingopyxis, on the basis of phylogenetic and chemotaxonomic analyses. Int J Syst Evol Microbiol 51, 1405-1417.

Thompson, J. D., Higgins, D. G. \& Gibson, T. J. (1994). CLUSTAL W: improving the sensitivity of progressive multiple sequence alignment through sequence weighting, position-specific gap penalties and weight matrix choice. Nucleic Acids Res 22, 4673-4680.

Wittich, R. M., Wilkes, H., Sinnwell, V., Francke, W. \& Fortnagel, P. (1992). Metabolism of dibenzo-p-dioxin by Sphingomonas sp. strain RW1. Appl Environ Microbiol 58, 1005-1010.

Yabuuchi, E. \& Ohyama, A. (1972). Characterization of "pyomelanin"-producing strains of Pseudomonas aeruginosa. Int J Syst Bacteriol 22, 53-64.

Yabuuchi, E., Yano, I., Oyaizu, H., Hashimoto, Y., Ezaki, T. \& Yamamoto, H. (1990). Proposals of Sphingomonas paucimobilis gen. nov. and comb. nov., Sphingomonas parapaucimobilis sp. nov., Sphingomonas yanoikuyae sp. nov., Sphingomonas adhaesiva sp. nov., Sphingomonas capsulata comb. nov., and two genospecies of the genus Sphingomonas. Microbiol Immunol 34, 99-119.

Yabuuchi, E., Kosako, Y., Yano, I., Hotta, H. \& Nishiuchi, Y. (1995). Transfer of two Burkholderia and an Alcaligenes species to Ralstonia gen. nov.: proposal of Ralstonia pickettii (Ralston, Palleroni and Doudoroff 1973) comb. nov., Ralstonia solanacearum (Smith 1896) comb. nov. and Ralstonia eutropha (Davis 1969) comb. nov. Microbiol Immunol 39, 897-904

Yabuuchi, E., Kosako, Y., Naka, T., Suzuki, S. \& Yano, I. (1999). Proposal of Sphingomonas suberifaciens (van Bruggen, Jochimsen and Brown 1990) comb. nov., Sphingomonas natatoria (Sly 1985) comb. nov., Sphingomonas ursincola (Yurkov et al. 1997) comb. nov., and emendation of the genus Sphingomonas. Microbiol Immunol 43, 339-349.

Yabuuchi, E., Yamamoto, H., Terakubo, S., Okamura, N., Naka, T., Fujiwara, N., Kobayashi, K., Kosako, Y. \& Hiraishi, A. (2001). Proposal of Sphingomonas wittichii sp. nov. for strain RW1 ${ }^{\mathrm{T}}$, known as a dibenzo-p-dioxin metabolizer. Int J Syst Evol Microbiol 51, 281-292.

Yurkov, V., Stackebrandt, E., Buss, O., Vermeglio, A., Gorlenko, V. \& Beatty, J. T. (1997). Reorganization of the genus Erythromicrobium: description of "Erythromicrobium sibiricum" as Sandaracinobacter sibiricus gen. nov., sp. nov., and of "Erythromicrobium ursincola" as Erythromonas ursincola gen. nov., sp. nov. Int J Syst Bacteriol 47, 1172-1178

Zipper, C., Nickel, K., Angst, W. \& Kohler, H.-P. E. (1996). Complete microbial degradation of both enantiomers of the chiral herbicide mecoprop [( $R S)$-2-(4-chloro-2-methylphenoxy)propionic acid] in an enantioselective manner by Sphingomonas herbicidovorans sp. nov. Appl Environ Microbiol 62, 4318-4322. 